\title{
Erratum to: On manifolds with multiple lens space fillings
}

\author{
Kenneth L. Baker • Brandy Guntel Doleshal • \\ Neil Hoffman
}

Published online: 9 December 2014

(C) Sociedad Matemática Mexicana 2014

\section{Erratum to: Bol. Soc. Mat. Mex. (2014) 20:405-447 DOI 10.1007/s40590-014-0016-8}

In our classification of non-hyperbolic knots in lens spaces with non-trivial Dehn surgeries to lens spaces, Theorem 13 of our work, we failed to acknowledge that the exteriors of the last three listed knot types may coincide. Since Theorem 13 only deals with non-hyperbolic manifolds much of the paper is unaffected. However, there is a family of toroidal manifolds with three lens space fillings of distance 1 that was initially overlooked and not included in Theorem 1. To accommodate this family and correct our arguments, we submit the following minor changes: the statement of Theorem 1 and the proof of Theorem 1 have a more comprehensive treatment of the toroidal case; the statement of Theorem 13 no longer claims that the cases are mutually exclusive; and the proof of Corollary 40 discusses the toroidal case in further detail.

The online version of the original article can be found under doi:10.1007/s40590-014-0016-8.

K. L. Baker $(\varangle)$

Department of Mathematics, University of Miami, PO Box 249085,

Coral Gables, FL 33124-4250, USA

e-mail:k.baker@math.miami.edu

B. G. Doleshal

Department of Mathematics and Statistics, Sam Houston State University, Box 2206, Huntsville, TX 77341, USA

e-mail: bdoleshal@shsu.edu

N. Hoffman

University of Melbourne, Parkville, VIC 3010, Australia

e-mail: nhoffman@ms.unimelb.edu.au 
Theorem 1 Let $M$ be a 3-manifold obtained by filling four boundary components of $M_{5}$. If $M$ admits three distinct lens space fillings, then either $M$ is the exterior of a trivial knot in a lens space, $M$ is the exterior of a torus knot in a lens space, $M$ is the exterior of the connected sum of a torus knot in $S^{3}$ with the unknotted core of $L(2,1)$, or $M$ with its lens space fillings are homeomorphic to a member of family $\mathcal{A}$ or $\mathcal{B}$.

Proof If $M$ is non-hyperbolic, then it follows from Theorem 13 that $M$ is the exterior of either a trivial knot, a torus knot in a lens space, or the connected sum of a torus knot in $S^{3}$ with the unknotted core of $L(2,1)$. If $M$ is hyperbolic then, using Martelli-Petronio-Roukema [3] as a starting point, Theorem 19 shows that $M$ must be homeomorphic to a filling of $M_{3}$. Theorem 22 then uses the Martelli-Petronio [2] catalog of exceptional fillings of $M_{3}$ to show $M$ with its lens space fillings belongs to family $\mathcal{A}$ or $\mathcal{B}$.

Remark The exterior of the connected sum of a $(p, q)$-torus knot in $S^{3}$ and the unknotted core of $L(2,1)$ can be obtained as $M_{5}(\infty, p / r,-s / q, 1 / 2)$ where $p s-r q=1$. The 0 -filling of the fifth component produces $L(2,1)$. The other two lens space fillings of this fifth component are +1 and $\infty$.

Theorem 13 Assume $K$ is a non-hyperbolic knot in a lens space with a non-trivial surgery yielding a lens space. Then $K$ is either

1. an unknot and surgery on $K$ along any slope produces a lens space,

2. a torus knot and surgery on $K$ along any slope distance 1 from $\lambda$ produces a lens space,

3. a $2 \lambda \pm \mu$-cable of a torus knot and \pm 1 surgery on $K$ with respect to the framing by the cabling annulus produces a lens space,

4. a $p \lambda+q \mu$-cable of a torus knot on which $p / q$-surgery produces $S^{3}$ and surgery on $K$ along the slope of the cabling annulus produces a lens space, or

5. the connected sum of a torus knot $T_{p, q}$ in $S^{3}$ and an unknot $U$ in $L(n, 1)$ and surgery of $K$ along the framing that arises in the connected sum from the framing $\lambda$ of $T_{p, q}$ and a framing on $U$ that gives $S^{3}$ by surgery produces a lens space.

The last two cases are surgery dual. Moreover, if the knot is not a torus knot, but has three lens space surgeries, then its exterior is toroidal and its surgeries correspond to each of the last three cases: it is a $2 \lambda \pm \mu$ cable of a $(p, q)$-torus knot in a lens space with a surgery to $S^{3}$ or dually the connected sum of a $(r, s)$-torus knot in $S^{3}$ with the unknot $U$ in $L(2,1)$. Otherwise the knot has only the stated non-trivial lens space surgery.

Proof of Corollary 40 By the Cyclic Surgery Theorem [1], if a manifold has three lens space fillings then either the manifold is reducible or a Seifert fibered space or the three fillings are mutually distance 1 . By Theorem 13 the only non-hyperbolic manifolds with at least three lens space fillings are the exteriors of torus knots and trivial knots, and a certain family of toroidal manifolds. If the lens space knots $K_{1}, K_{2}, K_{3}$ are duals to one another by distance 1 surgeries, then all three cannot be null-homologous. Thus, assuming they are not torus knots, up to homeomorphism and reindexing we may take $K_{1}$ to be the knot $K_{-3}^{-3+1 / k}$ or $K_{-2}^{-4+1 / k}$ for some integer $k \neq 0,1$. These 
have surgeries to the null-homologous knots $K_{\infty}^{-3+1 / k}$ and $K_{\infty}^{-4+1 / k}$ respectively as described in Theorem 35. From the proof of that theorem, the knots $K_{-3}^{-3+1 / k}$ do not have any other lens space surgery when $k \neq 0,1$. The same proof also shows that among the knots $K_{-2}^{1 / k, 1 / \ell}$, only the knot $K_{-2}^{-1,1}=K_{-2}^{1,-1}=K_{-2}^{-5}$ has the extra lens space surgery $K_{-1}^{-5}$. The manifold $K^{-5}=W(-5, \cdot)$ is the Figure Eight Knot Sister manifold.

\section{References}

1. Culler, M., Gordon, C.McA., Luecke, J., Shalen, P.B.: Dehn surgery on knots. Ann. Math. (2) 125(2), 237-300 (1987)

2. Martelli, B., Petronio, C.: Dehn filling of the "magic" 3-manifold. Commun. Anal. Geom. 14(5), 9691026 (2006)

3. Martelli, B., Petronio, C., Roukema, F.: Exceptional Dehn surgery on the minimally twisted five-chain link. Commun. Anal. Geom. 22(4), 689-735 (2014) 